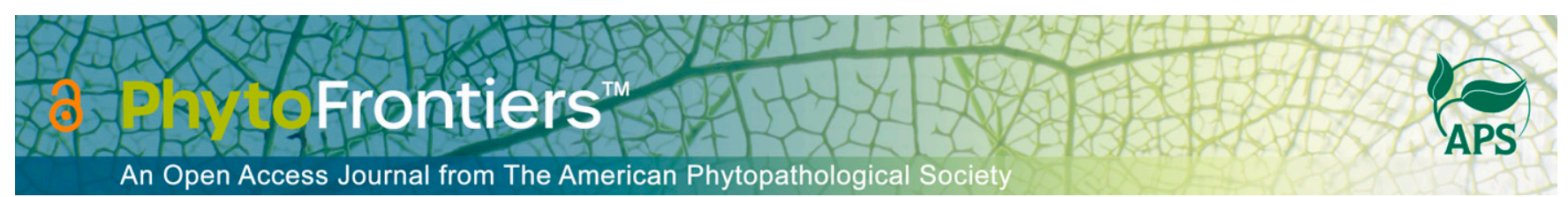

\title{
Research
}

\section{Interactions Between Delia platura and Erwinia amylovora Associated with Insect-Mediated Transmission of Shoot Blight}

\author{
Matthew Boucher ${ }^{1,+}$ (D) $\mid$ Rowan Collins $^{1} \mid$ Kayli Harling $^{1} \mid$ Gabrielle Brind'Amour $^{1} \mid{\text { Kerik } \text { Cox }^{2}(\mathbb{D})}$ | \\ Greg Loeb ${ }^{1} \mid$
}

${ }^{1}$ Department of Entomology, Cornell AgriTech, New York State Agricultural Experiment Station, Cornell University, Geneva, NY 14456

2 Section of Plant Pathology and Plant Microbe Biology, School of Integrated Plant Sciences, Cornell AgriTech, New York State Agricultura Experiment Station, Geneva, NY 14456

† Corresponding author: M. T. Boucher; mzb6294@ psu.edu

Accepted for publication 7 October 2020.

\begin{abstract}
Erwinia amylovora is a bacterial pathogen of rosaceous plants that can be devastating to commercial apple and pear production worldwide. Exopolysaccharide (EPS) production is essential for pathogenicity, aiding in biofilm production, and plant defense protection. EPS also plays an epidemiological role in the form of bacterial ooze, which is generated when $E$. amylovora builds to such high levels within the parenchyma that it ruptures the plant epidermis and a mixture of bacteria encased in EPS exudes from the injury. Insects such as Delia platura feed on the ooze and become potential vectors in the process. The goal of this study was to investigate interactions between $D$. platura and $E$. amylovora to better understand how insects facilitate shoot blight. We demonstrate for the first time that $D$. platura can successfully transmit pathogen cells that initiate new infections in mechanically damaged apple shoots and that EPS aids in adherence of $E$. amylovora to the insect surface. We show that flies can carry the bacteria externally for at least 5 days and that they shed a constant daily rate. We also show that $E$. amylovora strains differing in virulence do not behave differently when associated with $D$. platura and that consumption of $E$. amylovora has no effect on insect survival. Our data demonstrate that flies can be efficient facilitators of new shoot blight infections, but the field conditions that make this type of transmission possible require further investigation.
\end{abstract}

Keywords: Diptera, Hemiptera, Hymenoptera, Erwinia amylovora, fire blight, pollinators, blossom blight, ooze, exopolysaccharide

Fire blight is a bacterial disease of rosaceous plants caused by Erwinia amylovora that leads to significant losses in apple and pear production worldwide (van der Zwet et al. 2012). The bacterium can colonize any natural opening or injury on the plant surface and infects every host tissue type (Norelli et al. 2003), spreading systemically throughout the plant via the intercellular spaces of the cortical parenchyma (Bogs et al. 1998; Malnoy 
et al. 2012; Momol et al. 1998; Slack et al. 2017). An important virulence factor required for colonization and systemic movement of E. amylovora is exopolysaccharide (EPS) production (Denny 1995; Koczan et al. 2009). Exopolysaccharides are bacterially produced sugars that serve various functions during infection (Nimtz et al. 1996). E. amylovora EPS has three known components: amylovoran (Goodman et al. 1974), which makes up the bulk of the EPS, and levan and cellulose, which are minor components that enhance virulence (Koczan et al. 2009). Amylovoran is key to E. amylovora biofilm formation in apple xylem vessels, and mutant E. amylovora lacking the genes responsible for amylovoran production do not form biofilms (Koczan et al. 2009). Biofilms form across the xylem lumen and block nutrient movement and water uptake, leading to wilting symptoms characteristic of fire blight (Bogs et al. 1998; Koczan et al. 2009; Sjulin and Beer 1978). Levan also plays a role in biofilm formation and is hypothesized to aid in bacterial localization to host tissues (Geier and Geider 1993; Koczan et al. 2009). Additionally, E. amylovora EPS has been hypothesized to protect the bacterium from recognition by host plant defenses (Denny 1995; Geider 2004; Geier and Geider 1993). Multiple mechanisms have been proposed to explain this recognition phenomenon, including sequestration of toxic plant compounds and inhibition of contact between hypersensitive responseinducing harpins on the E. amylovora surface and plant cells (Denny 1995).

In addition to their role in virulence, EPSs produced by $E$. amylovora serve an important epidemiological function (Slack et al. 2017). The clogging of xylem vessels via bacterial and EPS aggregation leads to the rupture of xylem walls, allowing bacteria to colonize the parenchyma (Bogs et al. 1998). Continued $E$. amylovora growth and EPS production in the parenchyma lead to the buildup of a bacterial mass encased in EPS that creates pressure on the surrounding plant tissue (Slack et al. 2017). This pressure buildup ultimately causes a rupture of the plant epidermis through which bacteria encased in the EPS matrix exude (Slack et al. 2017). Epidemiologically, this ooze serves as primary and secondary inoculum from the early spring through midsummer, at which time new shoots set terminal buds and green tissue lignifies (Slack et al. 2017). Ooze enhances the longterm survival of bacteria encased in it because the bacteria are protected from environmental risks such as desiccation and ultraviolet radiation (Hildebrand 1939). Additionally, high E. amylovora populations in ooze allow for significant bacterial mortality without compromising the likelihood of infectivity following dispersal (Slack et al. 2017).

Insects, especially various species of dipterans, have been observed walking and feeding on ooze, and dissemination of $E$. amylovora from ooze has been identified as a pathway for disease transmission (Boucher et al. 2019; Parker 1936; Slack et al. 2017; Stewart and Leonard 1915, 1916). Despite early skepticism over the materiality of this pathway (Stewart and Leonard 1916), recent research indicates that it may be an important driver of $E$. amylovora transmission (Boucher et al. 2019; Ordax et al. 2015; Slack et al. 2017). Transmission is hypothesized to occur when flies acquire the bacteria while feeding on bacterial ooze and mechanically transmit it to plant surfaces (Boucher et al. 2019; Slack et al. 2017; Stewart and Leonard 1915). The mechanical nature of this mechanism was the primary source of skepticism over its relevance because the types of flies observed feeding on ooze do not generally cause damage to the plants (Stewart and Leonard 1916). This led researchers to believe that E. amylovora cells had no viable infection court to colonize when transmitted via this pathway, resulting in a dead end (Stewart and Leonard 1916). However, Slack et al. (2017) proposed that the high bacterial titers in ooze allowed insects to acquire enough $E$. amylovora to continuously disseminate it to each surface landed on before ultimately disseminating bacteria to the surface of a viable host. Boucher et al. (2019) confirmed that flies can acquire high titers of bacteria but did not evaluate how long insects could retain the bacteria, or if the insects could initiate a successful infection in plants with available entry courts.

Although the EPS that constitutes ooze and its contribution to virulence are well characterized (Koczan et al. 2009), relatively little is known regarding the parameters that dictate the epidemiological success of the EPS-insect transmission pathway. Components of this transmission pathway were demonstrated previously (Boucher et al. 2019; Ordax et al. 2015), but infection and symptom development have yet to be shown. Furthermore, rain can disperse E. amylovora from ooze, creating epiphytic bacterial populations on plant surfaces (Miller 1972), and it is unknown whether these populations can be acquired and transmitted by insects. Thus, one objective of this study was to determine whether nonpestiferous Diptera common to pome fruit orchards can initiate E. amylovora infections by disseminating bacteria to previously damaged plants. To gauge the relevance of epiphytic E. amylovora populations to insect-mediated transmission, we compared the incidence and severity of disease when D. platura acquired E. amylovora from ooze or from a contaminated surface lacking ooze. We conducted these experiments in a laboratory setting using Delia platura, a frequent insect visitor to ooze that is not known to cause damage to plants as an adult (Miller and McClanahan 1960).

Additionally, we evaluated various microbial dynamics relevant to insect-mediated transmission, including E. amylovora retention by $D$. platura, insect survival following $E$. amylovora ingestion, and differences in dynamics by bacterial strain. Historically, researchers have focused on external insect contamination as the main source of potential inoculum (van der Zwet and Keil 1979), but recent evidence suggests $E$. amylovora can survive gut passage and be defecated or regurgitated (Boucher et al. 2019; Ordax et al. 2015), which could extend the window of infectivity. Furthermore, survival of E. amylovora through the insect gut might indicate that the bacterium can use insects as alternative hosts, which has been demonstrated in related pathosystems (Correa et al. 2012; Grenier et al. 2006; Nadarasah and Stavrinides 2011). It is unknown whether E. amylovora uses insects as alternative hosts, and testing insect survival after ingestion of E. amylovora provides initial evidence as to whether this is the case. Finally, comparing dynamics between E. amylovora strains helps establish the generality of our findings and assess the effect of strain virulence on D. platura-E. amylovora interactions (Norelli et al. 1984), because it is unknown whether difference in virulence to plants affects interactions with insects. Specifically, we measured (i) differences in bacterial retention in and on $D$. platura based on the above-mentioned acquisition types, (ii) the effect of E. amylovora ingestion and strain on fly survival, and (iii) differences in shedding and retention rates of two E. amylovora strains. Collectively, these metrics detail interactions between $D$. platura and E. amylovora crucial to successful transmission of the pathogen to a plant host.

\section{MATERIALS AND METHODS}

\section{D. platura colony maintenance}

The D. platura colony was started in the summer of 2018 with wild flies captured in strawberry low tunnel plantings at Cornell AgriTech in Geneva, NY. Flies were captured daily when wild populations were abundant and kept in $61 \times 61 \times 61-\mathrm{cm}$ 
BugDorms (catalog no. 1462W, BioQuip Products, Rancho Dominguez, CA) in a walk-in environmental growth chamber with a $16-\mathrm{h} / 8$-h day/night cycle at $24^{\circ} \mathrm{C}$ and $50 \%$ relative humidity. Adult flies were maintained as described previously (Webb and Eckenrode 1978). Briefly, a 125-ml Erlenmeyer flask was filled with water, sealed with Parafilm, and then threaded with dental wick to provide a water source. A dry diet consisting of 10 parts skim milk powder, 10 parts table sugar, one part brewer's yeast, and one part soy peptone was provided in an open Petri dish. An extra Petri dish filled with brewer's yeast was provided for additional protein (Webb and Eckenrode 1978). Diet and water sources were replaced weekly.

An oviposition bin was created from two rectangular, 1.9-liter plastic containers. The lower container was filled to a depth of about $2.5 \mathrm{~cm}$ with water, and the upper container was punctured at its center and threaded with dental wick. The upper chamber was nested in the lower chamber, so the dental wick was in constant contact with the water and filled with about 1.2 liters of greenhouse sand. Fifty lima beans were pushed about an inch deep into the sand, and $4 \mathrm{ml}$ of meat and bone meal fertilizer (Keystone Mills, Romulus, NY) was layered on the surface. The water in the lower chamber kept sand moist, allowing beans to germinate, which stimulated D. platura oviposition. Moisture also prevented larval desiccation during development (Webb and Eckenrode 1978). Oviposition bins were transferred twice weekly to a development cage, where five to 10 additional beans and an additional layer of meat and bone meal were added. Larvae in oviposition bins developed for 2 weeks, after which most had pupated.

Pupae were initially separated from sand and plant debris by slowly adding room temperature water to the oviposition bin and then pouring the contents of the bin through a two-tiered soil sieve, with a $12.5-\mathrm{mm}$ mesh sieve placed above a $0.250-\mathrm{mm}, 60-$ mesh sieve to separate pupae from sand and plant debris. To maintain cleanliness, pupae were then floated in a bin of warm water and skimmed from the water surface (or removed from the bottom of the bin with butterfly forceps if they sank) to separate pupae from smaller plant debris. Pupae were dried on a paper towel, weighed, and placed onto the surface of an emergence bin. Emergence bins consisted of a 47-ml plastic deli cup, punctured at the center of the base and threaded with dental wick and filled with about $30 \mathrm{ml}$ of sand. This deli cup was nested in a $95-\mathrm{ml}$ deli cup filled $2.5 \mathrm{~cm}$ high with water so the dental wick was in constant contact with the water. The nested cups kept sand moist and prevented the pupae from desiccation. Emergence bins were kept in an emergence cage with water and dry diet as described above. Newly emerged flies were counted and transferred from the emergence cage to the main colony cages daily and would begin mating and laying eggs in oviposition bins at around 10 days postemergence. Dead flies were removed from main colony cages daily, and cages were replaced monthly to ensure cleanliness.

\section{E. amylovora strains}

Two strains were used in this study. Strain Ea273 (ATCC 49946) is a well-described strain isolated from apples in New York (Malus domestica cv. Rhode Island Greening) (Sebaihia et al. 2010). This strain was used in every experiment. Strain Ea266 is a highly virulent strain relative to Ea273 and was isolated from apple (cv. Rhode Island Greening) in Ontario, Canada (Norelli et al. 1984). This strain was used in shedding, survival, and internal load assays. Presumptive positive samples in all experiments were validated by growing individual colonies in Luria-Bertani (LB) broth overnight, clipping the center of the youngest leaf on an apple sapling shoot with scissors dipped in the culture, and observing until symptoms emerged for a maximum of 10 days.

\section{Apple sapling maintenance}

Brookfield Gala scions (M. domestica) approximately $0.6 \mathrm{~cm}$ in diameter bench grafted onto EMLA 26 rootstock (Schlabach Nursery, Medina, NY) were potted into $15-\mathrm{cm}$ square pots with a 3:1 mixture of LM-3 All Purpose Mix (Lambert, Quebec, Canada) and greenhouse sand. Plants were transferred to a greenhouse with $14-\mathrm{h} / 10$-h day/night cycle, with $22^{\circ} \mathrm{C}$ daytime temperature and $18^{\circ} \mathrm{C}$ nighttime temperature. Plants were watered as needed, and rootstock growth and blooms were removed by hand to promote shoot growth in the scion. We allowed three shoots to grow out of the scion, and any additional shoots were removed by hand. Hypoaspis spp. (Biobest Group, Westerlo, Belgium) were added to plants weekly to control for thrips. Plants were maintained in the greenhouse for 3 weeks before use in bioassays.

\section{Acquisition treatments}

Prior to use in experiments, flies were exposed to E. amylovora overnight (roughly $18 \mathrm{~h}$ ) in acquisition vials. Acquisition vials were made in two different ways as described below, one with $E$. amylovora available from simulated bacterial ooze (ooze acquisition) and the other with pure E. amylovora available from a filter disk (filter disk acquisition).

Simulated ooze vials were used to simulate fly feeding on bacterial ooze and were made by pipetting $6 \mathrm{ml}$ of CrosseGoodman (CG) medium into individual sterile plastic vials roughly $70 \mathrm{~mm}$ tall and $23 \mathrm{~mm}$ in diameter (Crosse and Goodman 1973). CG medium is selective for E. amylovora, and colonies present a distinct cratering morphology (Crosse and Goodman 1973). E. amylovora strain Ea273 was grown in LB broth for $8 \mathrm{~h}$ (approximating $6 \times 10^{8} \mathrm{CFU} / \mathrm{ml}$ ) at $28^{\circ} \mathrm{C}$ in a shake incubator, and $50 \mathrm{ml}$ of the resulting mixture was pipetted onto the surface of the CG medium in the plastic vials. Bacteria were spread on the medium surface by gently rotating, and vials were plugged with sterile paper towels to protect from contamination and then incubated in an environmental chamber (model I-30BL; Percival Scientific, Perry, IA) at $28^{\circ} \mathrm{C}$ for $48 \mathrm{~h}$. After $48 \mathrm{~h}$, a film formed across the surface of the medium with the same consistency and chemical makeup as bacterial ooze observed in the field (Bellemann et al. 1994; Nimtz et al. 1996). One- to 7-day-old flies were starved for $5 \mathrm{~h}$, and then 10 to 15 flies were added per vial. Vials were plugged with cotton and laid on their side in an environmental walk-in chamber with conditions matching those for colony maintenance. After overnight exposure, all flies were released into a single BugDorm cage (the holding cage) to randomize the flies used in experiments.

Filter disk vials were used to simulate epiphytic populations of E. amylovora that a fly may encounter (McManus and Jones 1994; Miller 1972), or as a standard testing acquisition method in survival and shedding assays (Chakrabarti et al. 2012). These vials were prepared by pipetting $6 \mathrm{ml}$ of $5 \%$ (w/v) sugar agar into individual sterile plastic vials. Sugar agar was made in batches, boiling $1,000 \mathrm{ml}$ of autoclaved $\mathrm{dH}_{2} \mathrm{O}$ before thoroughly mixing in $9 \mathrm{~g}$ of agar and $50 \mathrm{~g}$ of sugar. The mixture was simmered for $15 \mathrm{~min}$ and allowed to cool in a beaker before being distributed to vials (Siva-Jothy et al. 2018). Bacteria were prepared as described above, and $1 \mathrm{ml}$ of broth was aliquoted into 1.5 -ml centrifuge tubes and spun in a microcentrifuge at $4^{\circ} \mathrm{C}$ for $15 \mathrm{~min}$ at $2,500 \times g$ to pellet the bacteria. The supernatant was removed, and the pellet 
was resuspended in $1 \mathrm{ml}$ of 5\% sucrose solution (approximating $6 \times 10^{8} \mathrm{CFU} / \mathrm{ml}$ ). One hundred microliters of bacteria suspended in sucrose was pipetted onto 23 -mm filter disks and allowed to dry. Filter disks were placed on the surface of the solidified sugar agar using sterile forceps, and 1- to 7-day-old flies were prepared as described above.

\section{Transmission of E. amylovora by D. platura}

Three-week-old saplings were removed from the greenhouse, and two of the three shoots were removed from each sapling by hand. A sterilized 5-mm hole punch was used to make four wounds in each of the four youngest leaves of the shoot to simulate tissue damage and create wounds necessary for $E$. amylovora infection. Thirty-five inoculated flies were removed from the holding cage and released into a 2-liter paint strainer bag (W. W. Grainger, Lake Forest, IL) containing a moist piece of dental wick as a water source for the flies. The strainer bag was secured onto the sapling shoot so it covered only the damaged leaves and sealed at the open end with a twist tie. The strainer bag was adjusted by hand to ensure that the weight of the bag did not bend and snap the young shoot. Controls were treated as described above, but instead of being bagged with flies, the youngest leaf of each control was clipped at its center with scissors dipped in (i) an 8-h E. amylovora culture for positive controls or (ii) sterile $\mathrm{dH}_{2} \mathrm{O}$ for negative controls.

Saplings from all treatments were transferred to a mist chamber roughly $4.5 \times 4.5 \mathrm{~m}$ in size, arranged about $0.5 \mathrm{~m}$ apart from each other to avoid cross contamination, and held at $24^{\circ} \mathrm{C}$ with flies bagged to the plants for a 48 -h transmission period. During the transmission period, mist was turned on for $2 \mathrm{~h}$ each day to maintain a relative humidity of roughly $80 \%$. After the transmission period, strainer bags were removed from the plants and flies were discarded. Plants were incubated in the mist chamber for an additional 8 days, with the mist on for 3 to $4 \mathrm{~h}$ per day to maintain 85 to $100 \%$ relative humidity. After 10 days, plants were removed from the chamber for disease assessments. Two measurements were taken: (i) disease incidence, a binomial measure of whether symptoms were present; and (ii) severity, in which the length of the fire blight lesion was measured in proportion to the total length of the shoot.

We tested 50 total saplings across acquisition treatments (30 for E. amylovora ooze and 20 for E. amylovora culture on a filter disk), setting up a maximum of 25 trees per week to avoid overcrowding the mist chamber and to prevent cross contamination. For the ooze treatment, 10 replicates were tested with five positive controls and five negative controls, which was repeated for a second round after the disease assessment for the first round was completed. The same blocking pattern was replicated for the filter disk treatment, but we added five ooze replicates as an additional positive control in each round. Due to this blocking pattern, the ooze treatment is the only treatment with replicates in all blocks. A generalized linear model with binomial distribution analysis was fit to disease incidence in ooze replicates by block. The outcome of this modeling indicated no significant $(P>0.05)$ blocking effect, so all ooze replicates were used in the final analysis. If negative controls exhibited positive symptoms in a given round, all replicates in that round were composted and the experiment was restarted. However, only one negative control exhibited symptoms in all experiments, and this was likely due to transportation of negative control plants on the same tray as positive control plants. All positive controls developed symptoms, and severity of positive controls was compared with severity of acquisition treatments.

The initial dose of E. amylovora in 30 flies subjected to the ooze acquisition treatment and 20 flies subjected to the filter disk acquisition treatment was determined as described in the next section. All flies subjected to ooze acquisition were tested for initial dose using flies from the first block of the ooze transmission experiment. Flies subjected to filter disk acquisition were tested for initial dose in two rounds of 10 flies each, with each round corresponding to one of the two blocks of the filter disk transmission experiment. A Wilcoxon rank-sum test showed no differences in initial dose between the two blocks of filter disk flies, so data points were aggregated for comparison with ooze acquisition method flies.

Differences in incidence by acquisition treatments were analyzed in $\mathrm{R}$ using the lme4 package with a generalized linear mixed-effects model and a binomial distribution (Bates et al. 2015; R Core Team 2019). Treatment (ooze or filter disk) was the only fixed effect, and block was the only random effect. The model was fit by maximum likelihood, and direct comparisons between treatments were made using the estimated marginal means with the emmeans package in $\mathrm{R}$ (Lenth 2020). Differences in severity by acquisition treatments (ooze, filter disk, and control) were analyzed using a linear mixed-effects model with treatment as a fixed effect and block as a random effect. The model was fit by restricted maximum likelihood, and pairwise comparisons within treatment were made using the Tukey honestly significant difference (HSD) method in the emmeans package (Lenth 2020). Differences in initial dose between acquisition treatments (ooze and filter disk) were analyzed using a Wilcoxon rank-sum test for the $\log _{10}(x+1)$ transformed CFU values in $\mathrm{R}$ ( $\mathrm{R}$ Core Team 2019). Data were visualized using the ggplot2 package in R (Wickham 2016).

\section{Shedding of $E$. amylovora by D. platura}

One- to 7-day-old flies were exposed to either E. amylovora strain Ea273, Ea266, or a 5\% sucrose control using filter disk acquisition vials described above. After acquisition, all exposed flies were released into a single cage for each treatment, and 10 flies for each treatment were captured in individual $1.5-\mathrm{ml}$ vials supplied with $125 \mu$ l of sugar agar as a food source. Flies were transferred to fresh vials every $24 \mathrm{~h}$ for 4 days. The titer of viable E. amylovora shed was estimated by rinsing the vial with $1 \mathrm{ml}$ of $1 \times$ phosphate-buffered saline (PBS) for $20 \mathrm{~s}$ and serial diluting the rinse for culturing. Twenty microliters of the three most dilute for each sample were drop plated onto CG medium plates (Crosse and Goodman 1973). Plates were sealed with Parafilm and incubated upside down at $28^{\circ} \mathrm{C}$ for $48 \mathrm{~h}$. CFU/ml was then calculated by counting the dilution that yielded roughly 30 to 300 colonies for each sample (Siva-Jothy et al. 2018). The minimum detection threshold for this assay was roughly $500 \mathrm{CFU} / \mathrm{ml}$; counts below this level were not detectable and considered zeroes. Flies were sexed after the 4-day testing period was completed to evaluate the effect of sex on shedding rate.

We tested 30 flies for each strain and the sucrose control for a total of 90 flies. This experiment was conducted across 3 weeks, with 10 flies for each of the three treatments tested per week. Data were analyzed in $\mathrm{R}$ using a repeated measures hurdle model to account for zero inflation using the glmmTMB package (Brooks et al. 2017). The count portion of the model was fit with a truncated negative binomial distribution, and the zero inflated portion of the model was fit as a logistic regression. The model investigated the effects of strain, day, and sex on (i) the presence or absence of bacteria shed by flies (zero inflated portion) and (ii) the total $\mathrm{CFU} / \mathrm{ml}$ of bacteria shed by flies (count portion). Replicate nested within block was included as a random effect in all model iterations for both portions of the model. Model portions were initially constructed with all factors and 
interactions and simplified in a nested fashion. Nested models were compared using the Akaike information criterion (AIC) via the bbmle package (Bolker and R Development Core Team 2020), and the most parsimonious model (lowest AIC score) was chosen for final analysis (Grab et al. 2018). The count portion of the model ultimately included the fixed effects of all three factors, and the zero inflated portion of the model ultimately included only the day postacquisition (DPA) as a fixed effect. Post hoc pairwise comparisons using the Tukey HSD method within and among treatments were made for both portions of the model using the estimated marginal means in the emmeans package (Lenth 2020). Data were visualized using the ggplot2 and emmeans packages in R (Lenth 2020; Wickham 2016).

\section{E. amylovora load dynamics on/in D. platura}

One- to 7-day-old flies were subject to three E. amylovora treatments: (i) Ea273 via filter disk acquisition; (ii) Ea273 via ooze acquisition; and (iii) Ea266 via filter disk acquisition. A negative control in which flies were exposed to $5 \%$ sucrose via filter disk acquisition was also included. One hundred fifty flies were exposed to each treatment and released as groups into cages in an environmental chamber with conditions matching those used for colony maintenance. Cages contained dry diet and a water source as described in the colony maintenance section, and flies were never exposed to E. amylovora after the initial acquisition phase. Immediately upon release, 10 flies from each cage were captured in individual sterile $1.5-\mathrm{ml}$ microcentrifuge tubes to determine the initial dose of bacteria acquired by the flies. For both Ea273 treatments, flies were subjected to external wash by adding $1 \mathrm{ml}$ of $1 \times$ PBS to the tube and mixing the fly for $30 \mathrm{~s}$ using a vortex (Thomson et al. 2017). The fly was removed from the wash with sterile forceps and transferred to a sterile 1.5$\mathrm{ml}$ microcentrifuge tube containing $100 \mu \mathrm{l}$ of $70 \%$ ethanol for $1 \mathrm{~min}$ for surface sterilization. The external wash was diluted, plated, and counted as described in the shedding assay section.

After insects were sterilized, the ethanol was removed from the tube with a pipette, and the sample was rinsed in $100 \mu$ of $1 \times$ PBS for 1 min. The PBS rinse was removed with a pipette and spot checked to ensure proper surface sterilization by plating methods described above. Flies were homogenized in $200 \mu \mathrm{l}$ of $1 \times$ PBS with autoclaved plastic pestles, and samples were serially diluted, plated, and counted as described above. Ea266 samples were not evaluated for external load and were processed as described above starting with surface sterilization. Sucrose samples were immediately homogenized in $200 \mu \mathrm{l}$ of $1 \times$ PBS and treated as described as a negative control. No viable E. amylovora was detected in sucrose controls.

The above procedure was repeated daily to measure depreciation of bacterial load over time. External load measurements for both Ea273 treatments were terminated after 5 days, and internal load measurements for Ea273 ooze treatments were also terminated after 5 days. Internal load measurements for Ea273 filter disk, Ea266 filter disk, and sucrose were terminated after 7 days. This procedure was repeated two additional times, resulting in 30 fly replicates per DPA per treatment.

The effect of E. amylovora location (internal or external), acquisition method (ooze or filter disk acquisition), and DPA (1 to 5) on CFU acquired by D. platura was analyzed using a hurdle model to account for zero inflation in the glmmTMB package in $\mathrm{R}$ (Brooks et al. 2017). The count portion of the model was fit using a truncated negative binomial, and the zero inflated portion was fit as a logistic regression. Model simplification and selection were conducted as described above, and all iterations of both model portions included replicate nested within block (when the experiment was run) as a random effect. Both model portions included fixed effects of all three factors and two-way interaction terms for treatment by location, treatment by day, and location by day. Initial dose (count for 0 DPA) was not included in this analysis because all samples were positive for flies subject to the ooze acquisition method, which led to a lack of variation necessary to compare probability of infection at 0 DPA between acquisition method treatments. Likewise, three-way interactions were excluded from the model selection process because all flies subject to the ooze acquisition method at 1 and 3 DPA were positive for E. amylovora, again leading to a lack of necessary variation to include the term. Post hoc pairwise comparisons were made for both portions of the model using the Tukey HSD method in the emmeans package (Lenth 2020).

The effect of E. amylovora strain (Ea273 or Ea266) and DPA (0 to 7 ) on the internal load of E. amylovora in D. platura was analyzed using a hurdle model to account for zero inflation in the glmmTMB package in $\mathrm{R}$ (Brooks et al. 2017). The count portion of the model was fit using a truncated negative binomial, and the zero inflated portion was fit as a logistic regression. Model simplification and selection were conducted as described above, and block was included as a random effect in all model iterations. The final model included DPA as the only fixed effect in the zero inflated portion, and the count portion included strain, DPA, and a strain by DPA interaction as fixed effects. Post hoc pairwise comparisons were made using the Tukey HSD method as described above.

\section{Survival of $D$. platura after infection with $E$. amylovora}

One- to 7-day-old flies were subject to three infection treatments via filter disk acquisition: (i) Ea273, (ii) Ea266, and (iii) sucrose control. One hundred twenty to 130 flies were inoculated for each treatment and released into one cage per treatment, each containing dry diet and a water source (Webb and Eckenrode 1978). Cages were held in an environmental chamber with conditions matching those used for colony maintenance. Ten flies were immediately removed from the cage to determine initial dose as described in the previous section and to ensure no contamination in the sucrose control. Each day for 29 days, cages were checked for dead flies, which were removed, counted, and discarded. Diet and water were replaced every 3 days to ensure flies did not reacquire bacteria. Cages were rotated to a new spot in the environmental chamber every day to control for possible location effects. After 29 days, the remaining live flies were counted, and the proportion of flies alive on each day was determined using the sum of the flies remaining at the end of the test period and the total number of dead flies over 29 days. This experiment was replicated two additional times, and data were pooled and analyzed with the survival and survminer packages in $\mathrm{R}$ using a Cox proportional hazards model that included the effects of treatment and replicate on fly survival (Kassambara et al. 2019; Therneau 2020).

\section{RESULTS}

\section{Transmission of $E$. amylovora by $D$. platura}

D. platura that acquired E. amylovora via ooze successfully initiated infections in $95 \%$ of cases, whereas D. platura that acquired E. amylovora via filter disks successfully initiated infections in $30 \%$ of cases (Fig. 1). Based on differences in estimated marginal means, flies that acquired E. amylovora via ooze were significantly more likely to successfully initiate an infection relative to flies that acquired E. amylovora via filter disks $(\mathrm{Z}$ ratio $=-3.448, P<0.001)$. 
Mean severity in apple saplings exposed to flies subjected to ooze acquisition was $0.77 / 1.00$, compared with $0.25 / 1.00$ in apple saplings exposed to flies subjected to filter disk acquisition (Fig. 2). Positive control plants had a mean severity of 0.97/1.00. Severity was significantly higher in control plants relative to plants exposed to both acquisition treatments (ooze acquisition: $\mathrm{t}$ ratio $=2.569, P=0.033$; filter disk acquisition: $\mathrm{t}$ ratio $=6.977$, $P<0.0001)$. Severity was statistically higher in plants subjected to ooze acquisition flies compared with plants subjected to filter disk acquisition flies ( $\mathrm{t}$ ratio $=-4.180, P<0.0001$ ).

The initial dose of flies subjected to ooze acquisition ranged from 7.3 to $8.6 \log _{10} \mathrm{CFU} / \mathrm{ml}$ with a median value of $8.2 \log _{10}$ $\mathrm{CFU} / \mathrm{ml}$, whereas the initial dose of flies subjected to filter disk acquisition ranged from 5.7 to $7.3 \log _{10} \mathrm{CFU} / \mathrm{ml}$ with a median value of $6.7 \log _{10} \mathrm{CFU} / \mathrm{ml}$ (W $=600, P<0.0001$, Fig. 3).

\section{Shedding of E. amylovora by D. platura}

There was a main effect of day on the predicted probability that a fly will shed E. amylovora, which declined gradually over time. Flies were equally likely to shed bacteria 1 and 2 DPA ( $\mathrm{t}$ ratio = $1.754, P=0.299)$, with a significant drop in predicted probability

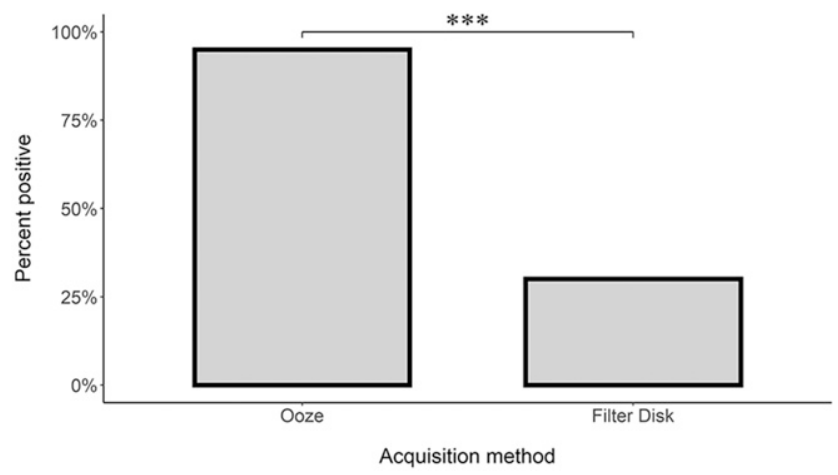

FIGURE 1

Incidence of Erwinia amylovora infection in Gala apple saplings when exposed to pathogen by 35 Delia platura that acquired bacteria through ooze or filter disks (no ooze). The two acquisition methods were meant to simulate possible sources of $E$. amylovora acquisition in the field, with ooze being the most readily available and filter disks simulating epiphytic or dispersed sources of $E$. amylovora. ${ }^{* * *}=P<0.0001$.

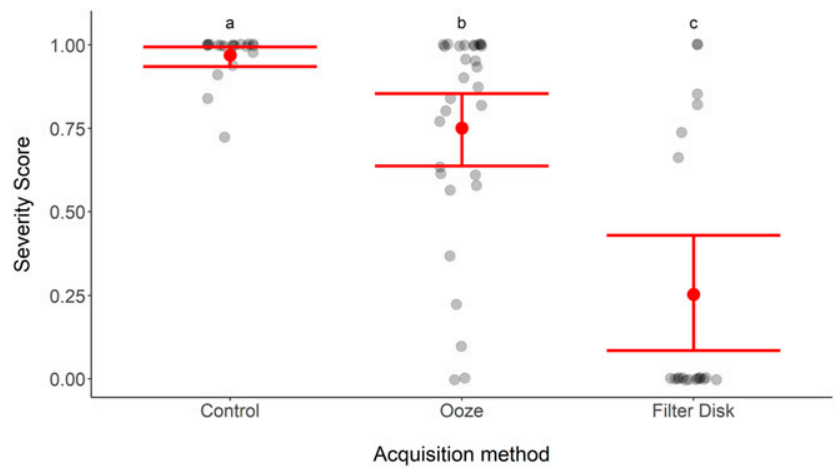

FIGURE 2

Mean $\pm 95 \%$ confidence interval disease severity in Gala apple saplings. Gray points represent individual severity scores for each replicate, and darker shading of those points indicates a greater accumulation of scores at that point. Letters above each factor level indicate significant differences from each other as determined by Tukey HSD adjusted pairwise comparisons. between 2 and 3 DPA (t ratio $=2.723, P=0.035$ ) and a nonsignificant drop in predicted probability between 3 and 4 DPA (t ratio $=1.871, P=0.244$ ) (Fig. 4). Flies had a significantly lower probability of shedding bacteria 3 and 4 DPA compared with 1 DPA (t ratio $=3.637, P=0.002$ and $\mathrm{t}$ ratio $=4.294, P=0.0002$, respectively) and significantly lower probability of shedding 4 DPA compared with 2 DPA (t ratio $=3.727, P=0.001$ ).

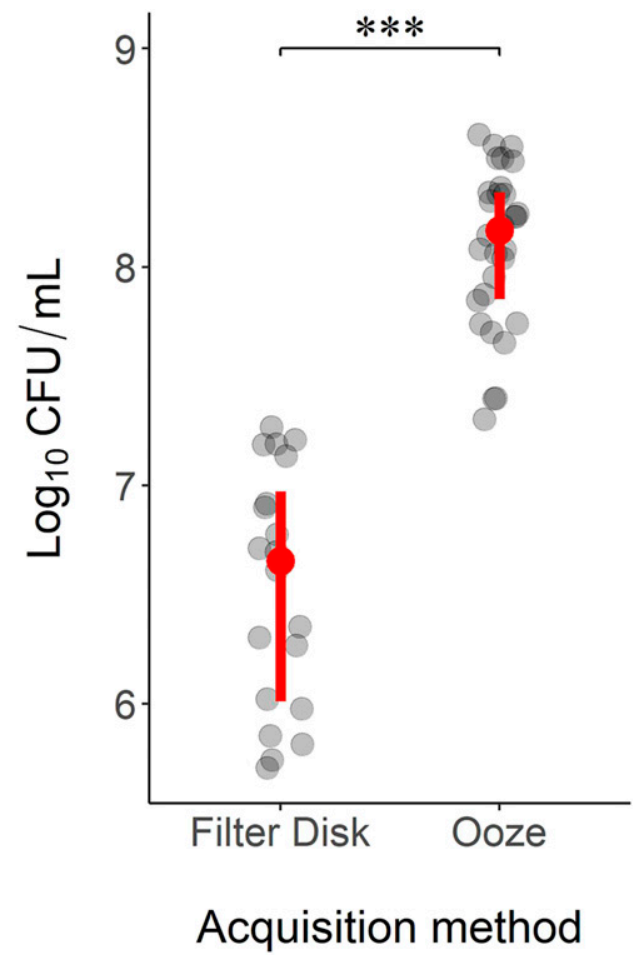

FIGURE 3

Median + interquartile range of initial dose in $\log _{10} \mathrm{CFU} / \mathrm{ml}$ between flies that acquired Erwinia amylovora from ooze and from filter disks. Gray points represent the $\log _{10} \mathrm{CFU} / \mathrm{ml}$ of tested individuals, and darker shading indicates accumulation of individuals at that point. Medians were compared using a Wilcoxon rank sum test, which showed that flies that acquired $E$. amylovora from ooze had significantly higher initial doses than flies that acquired $E$. amylovora from filter disks $(\mathrm{W}=600, P<0.0001) .{ }^{* * *}=P<0.0001$.

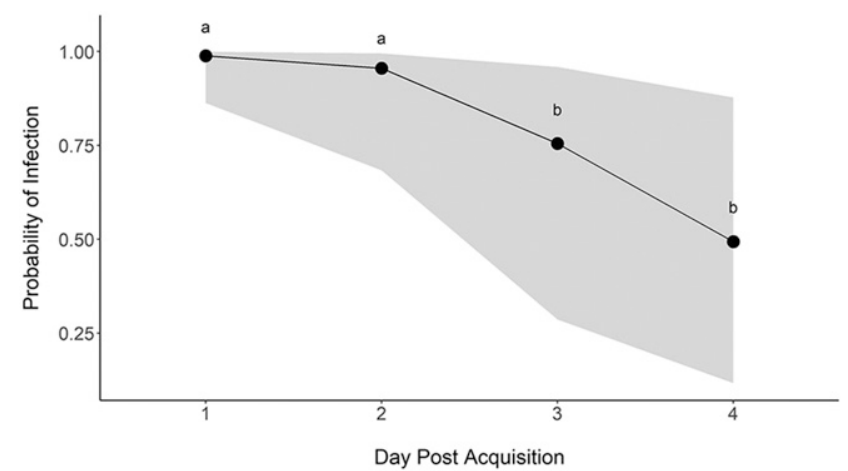

FIGURE 4

Predicted probability $\pm 95 \%$ confidence interval that a fly will shed Erwinia amylovora on a given day. Predicted probability gradually decreases over time, suggesting that flies are clearing acquired bacterial loads below detection thresholds as days postacquisition (DPA) increases. DPA is a strong predictor of bacterial shedding by flies. Letters above predicted probability values indicate which differences between probabilities are significant, with the same letter indicating nonsignificance. 


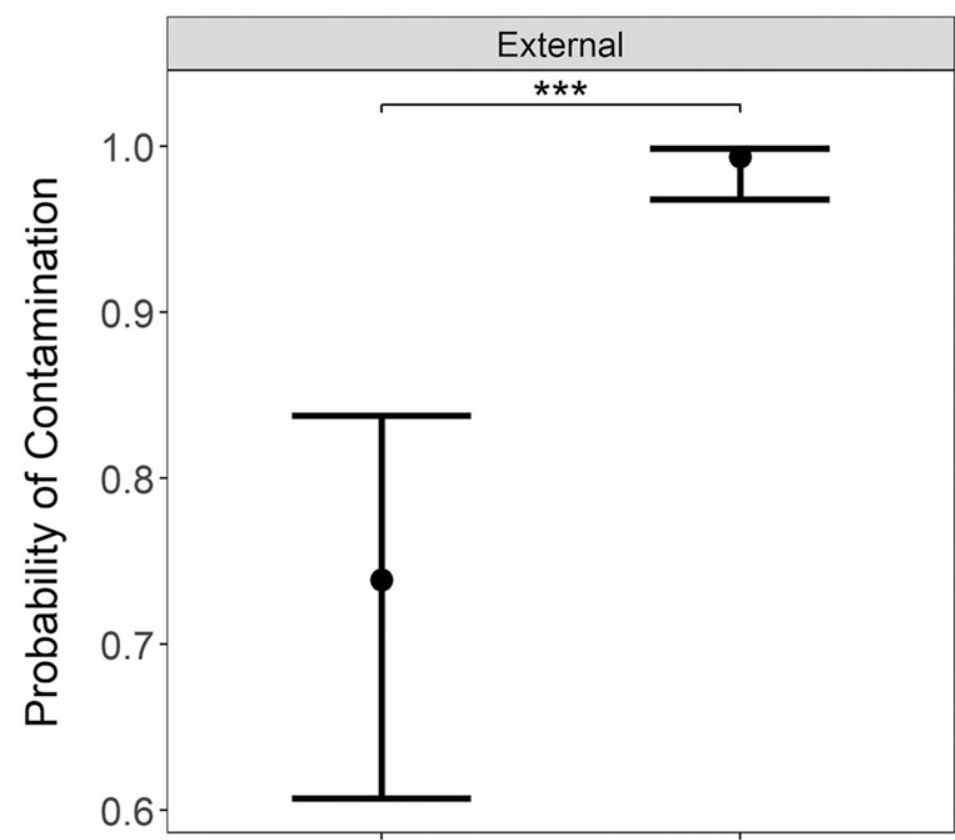

Filter Disk

Ooze

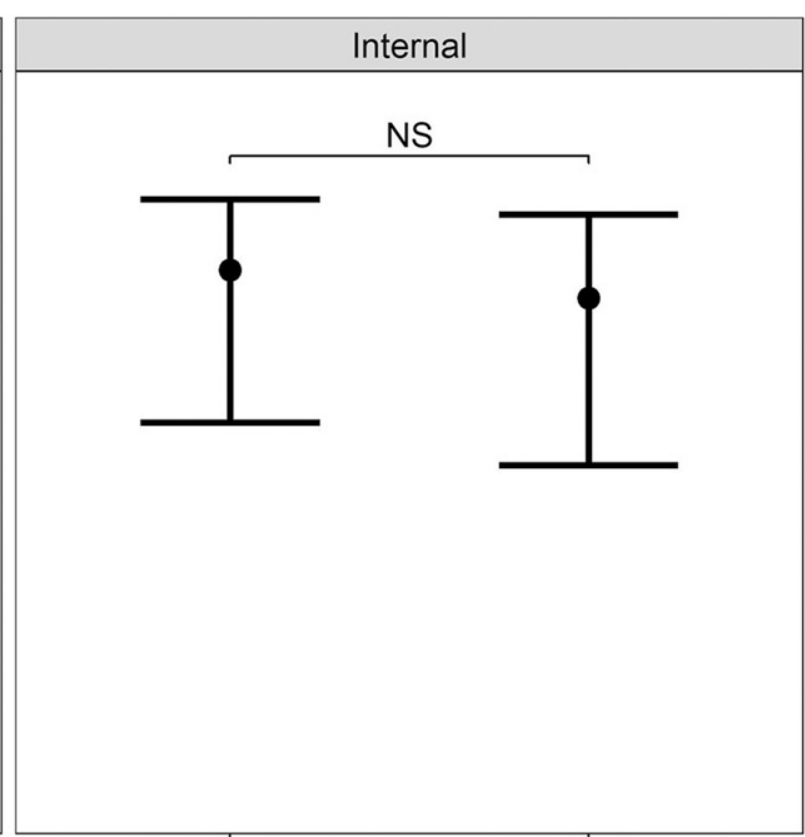

Filter Disk
Ooze

\section{Acquisition Method}

\section{FIGURE 5}

Predicted probability $\pm 95 \%$ confidence interval that a fly will be contaminated with Erwinia amylovora externally or internally based on acquisition method. Data compare the likelihood of a fly being contaminated when acquiring bacteria from filter disks or from ooze within location. ${ }^{* *}=P<0.0001$; and NS = not significant.
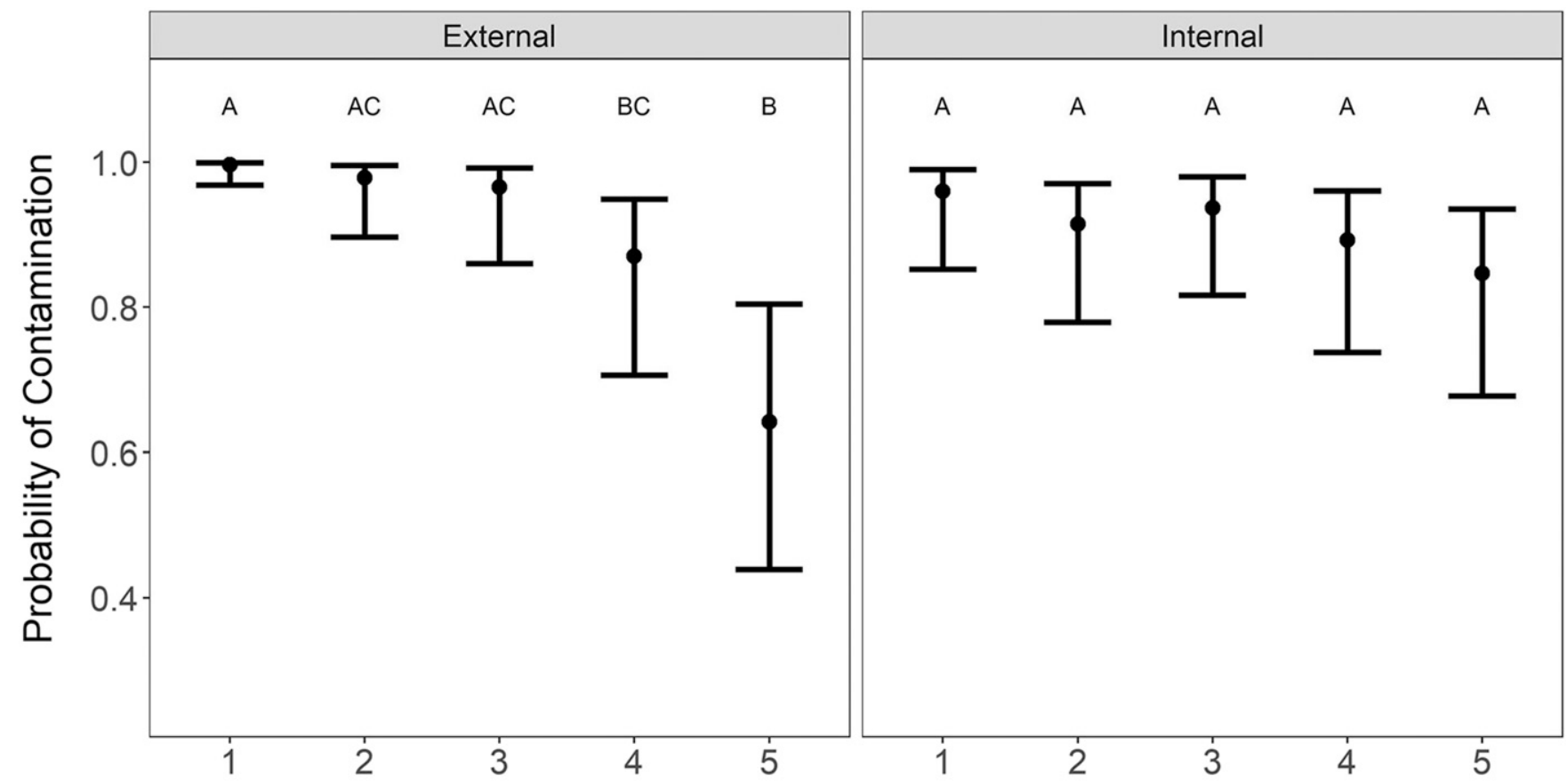

\section{Days Post Acquisition}

FIGURE 6

Predicted probability $\pm 95 \%$ confidence interval that a fly will be internally or externally contaminated with Erwinia amylovora by days postacquisition. The likelihood of external contamination decreased over time, whereas the likelihood of internal contamination remained constant over time. Letters above each point indicate significant differences within location as determined by Tukey HSD adjusted pairwise comparisons. 
Numerically, there was a slight decline in CFU counts of positive flies over time, but the difference in CFU between 1 and 4 DPA (the first and last days of testing) was not significant (t ratio $=2.081, P=0.164)$. There was no effect of $E$. amylovora strain on counts of bacteria shed by $D$. platura $(\mathrm{t}$ ratio $=0.260$, $P=0.795)$. There was no effect of sex on shed bacteria count (t ratio $=-1.566, P=0.119)$.

\section{E. amylovora load dynamics on/in D. platura}

There was a treatment by location interaction on the predicted probability of E. amylovora presence such that flies subjected to ooze acquisition were more likely to be externally contaminated relative to flies subjected to filter disk acquisition ( $\mathrm{t}$ ratio $=$ $-5.205, P<0.0001$ ), but flies subjected to either treatment were equally likely to be internally contaminated (t ratio $=0.494, P=$ 0.961 , Fig. 5). Flies subjected to filter disk acquisition were more likely to be contaminated internally relative to externally $(\mathrm{t}$ ratio $=$ $-3.749, P=0.001$ ), but flies subjected to ooze acquisition were more likely to be contaminated externally relative to internally (t ratio $=4.196, P<0.001)$.

Flies had a higher probability of external contamination relative to internal contamination at 1,2 , and 3 DPA, but a higher probability of internal contamination relative to external contamination at 4 and 5 DPA. None of the differences between internal and external contamination probability on a given day were significant (for pairwise comparisons across all days: $\mathrm{t}$ ratio $=-2.050$ to $2.737, P>0.05$ ). There was a decline in probability of external contamination over time, as evidenced by significant differences between the first day of testing and the fourth (t ratio $=3.493, P=0.018$ ) and fifth (t ratio $=4.400, P<$ 0.001 ) day of testing (Fig. 6). Flies were equally likely to be externally contaminated from 1 to 3 DPA (for pairwise comparisons of 1 to 3 DPA: $t$ ratio $=0.565$ to $2.149, P>0.05$ ), from 2 to $4 \mathrm{DPA}$ (for pairwise comparisons of 2 to $4 \mathrm{DPA}$ : $\mathrm{t}$ ratio $=0.565$ to $2.287, P>0.05$ ), and from 4 to 5 DPA (t ratio $=2.061, P=$ 0.56 , Fig. 6). Flies were equally likely to be internally contaminated across time, as evidenced by no statistical differences between any day (for pairwise comparisons across all days: $\mathrm{t}$ ratio $=-0.477$ to $1.912, P>0.05)$.

When location of bacteria was controlled for statistically, flies had a higher probability of E. amylovora contamination when subjected to ooze acquisition relative to filter disk acquisition at 1 DPA ( $\mathrm{t}$ ratio $=-3.234, P=0.042$ ), but flies were equally likely to be contaminated regardless of acquisition treatment on all other days (for pairwise comparisons at 2 to 5 DPA: $t$ ratio $=-2.908$ to $0.289, P>0.05)$. When subjected to filter disk acquisition, flies were equally likely to contain E. amylovora across all days (for pairwise comparisons across all days: $\mathrm{t}$ ratio $=-0.678$ to 2.026 , $P>0.05$ ), indicating a steady presence over time. When subjected to ooze acquisition, flies were equally likely to contain $E$. amylovora from 2 to $5 \mathrm{DPA}$ (for pairwise comparisons at 2 to 5 DPA: $t$ ratio $=0.531$ to $2.724, P>0.05$ ) and from 1 to $3 \mathrm{DPA}$ (for pairwise comparisons from 1 to $3 \mathrm{DPA}$ : $\mathrm{t}$ ratio $=0.647$ to 1.977 , $P>0.05)$ but were more likely to carry bacteria at $1 \mathrm{DPA}$ than at 4 DPA (t ratio $=3.445, P=0.022)$ and 5 DPA (t ratio $=3.681, P=$ 0.01 ), indicating a decline in the presence of bacteria over time.

Within acquisition treatment, internal bacterial counts were significantly higher than external bacterial counts in flies subjected to filter disk acquisition ( $\mathrm{t}$ ratio $=-18.880, P<0.0001$ ) and from ooze acquisition (t ratio $=-17.543, P<0.0001$, Fig. 7). This pattern was consistent on all days (for pairwise comparisons across all days: $\mathrm{t}$ ratio $=-12.684$ to $-9.367, P<0.0001)$. Internal E. amylovora counts between the two acquisition treatments were

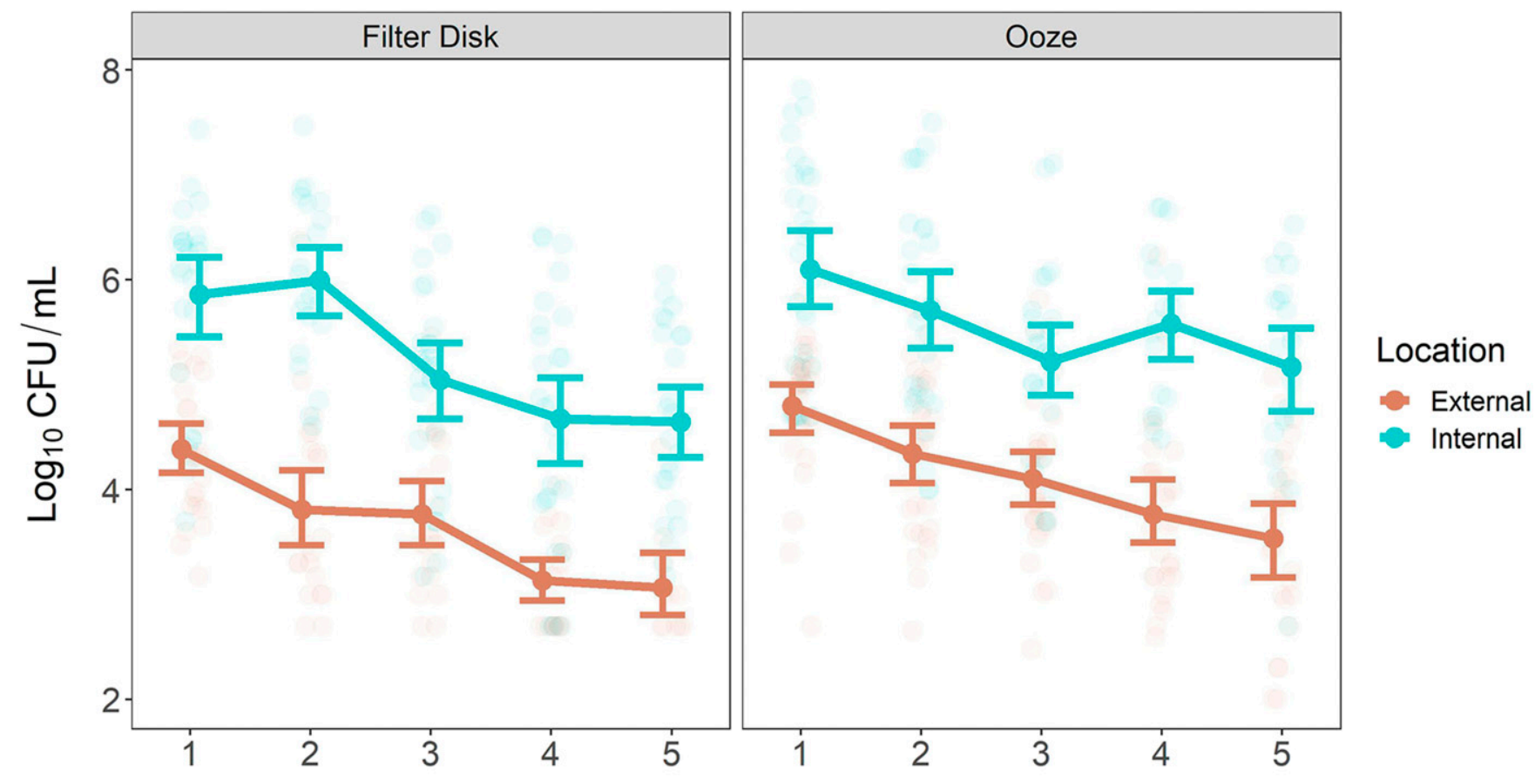

Days post acquisition

FIGURE 7

Mean $\pm 95 \%$ confidence interval of Erwinia amylovora load on internal and external surfaces of Delia platura in Log 10 CFU/ml separated by acquisition method. Shaded points show $\log _{10} \mathrm{CFU} / \mathrm{ml}$ counts of individual replicates, colored by location on $D$. platura. Data compare internal and external load within acquisition method. Internal loads were higher than external loads in both acquisition methods, and this pattern was consistent across all days as determined by Tukey HSD adjusted pairwise comparisons. 
significantly different such that flies subjected to ooze acquisition had higher overall counts relative to flies subjected to filter disk acquisition (t ratio $=-3.448, P=0.003$ ). External counts followed the same pattern ( $\mathrm{t}$ ratio $=-5.320, P<0.0001$ ).

External bacterial loads declined over time with significant differences at 1 and 3 DPA, 1 and 4 DPA, 1 and 5 DPA, 2 and 4 DPA, 2 and 5 DPA, 3 and 4 DPA, and 3 and 5 DPA (for pairwise comparisons of noted days: $t$ ratio $=3.412$ to $7.317, P<0.05$, Fig. 8). There were no significant differences at 1 and 2 DPA, 2 and 3 DPA, and 4 and 5 DPA (for pairwise comparisons of noted days: t ratio $=0.475$ to $2.437, P>0.05$ ). Internal bacterial loads declined over time such that 1 to 2 DPA were significantly different from 3 to 5 DPA (t ratio $=4.127$ to $7.028, P<0.05$ ), where the days within each group do not differ ( $\mathrm{t}$ ratio $=-0.280$ to 1.589 , $P>0.05$, Fig. 8).

Total bacterial loads varied between acquisition treatments at 4 DPA (t ratio $=-4.470, P<0.001$ ) and 5 DPA (t ratio $=-3.344$, $P<0.05$ ), with flies subjected to ooze acquisition carrying higher loads than flies subjected to filter disk acquisition. Numerically, total loads in flies subjected to ooze acquisition were higher than flies subjected to filter disk acquisition at 1 to 3 DPA, but the differences were not significant ( $\mathrm{t}$ ratio $=-2.180$ to $-0.977, P>$ $0.05)$. Total loads in flies subjected to filter disk acquisition did not vary on consecutive days ( $\mathrm{t}$ ratio $=0.484$ to $3.006, P>0.05$ ), but there was a significant difference between total load on the first and last day of testing ( $\mathrm{t}$ ratio $=6.833, P<0.0001$ ), indicating a decline in total load over time. The same pattern held for flies subjected to ooze acquisition (for differences on consecutive days: $\mathrm{t}$ ratio $=-0.284$ to $2.586, P>0.05$; for differences between first and last day of testing: $\mathrm{t}$ ratio $=5.869$, $P<0.0001)$.

There was no within-day effect of E. amylovora strain on the internal load of flies subjected to filter disk acquisition (for comparisons between strains within day: $\mathrm{t}$ ratio $=-2.816$ to 1.648, $P>0.05$ ). Strain Ea273 loads were stable over three distinct periods such that 0 to 2 DPA were not different from each other, 3 to 5 DPA were not different from each other, and 5 to 7 DPA were not different from each other (Fig. 9). Strain Ea266 internal loads were stable from 0 to 2 DPA before dropping at 3 DPA (Fig. 9). The mean internal load at 3 DPA was significantly different from mean internal load at 1 DPA but not significantly different from any other day. There was a numerical increase in internal load between 3 and 4 DPA, which was not significant, and internal load at 4 DPA was significantly different from 6 DPA but no other. At 5 DPA, internal load was significantly different from 0 to 2 DPA but not statistically different from all other days. At 6 DPA, internal load was significantly different from 0 to 2 DPA and 4 DPA but not significantly different from all other days. At 7 DPA, internal load was significantly different from 0 to 2 DPA but not different from 3 to 6 DPA.

\section{Survival of $D$. platura after infection with $E$. amylovora}

Although flies fed either strain Ea266 or strain Ea273 tended to die at slightly higher rates than flies fed sucrose, this was not statistically significant (Ea266: $\mathrm{z}=0.975, P=0.330 ; \mathrm{Ea} 273: \mathrm{z}=$ $1.260, P=0.208$, Fig. 10). There was a significant difference between replicates such that survivability in block 2 was significantly higher than the reference block (block 1$)(\mathrm{z}=-12.437$, $P<0.0001)$. Survivability between the reference block and block 3 did not differ $(\mathrm{z}=-1.758, P=0.079)$.

\section{DISCUSSION}

To our knowledge, we are the first to establish that $D$. platura can transmit E. amylovora to mechanically damaged shoots,

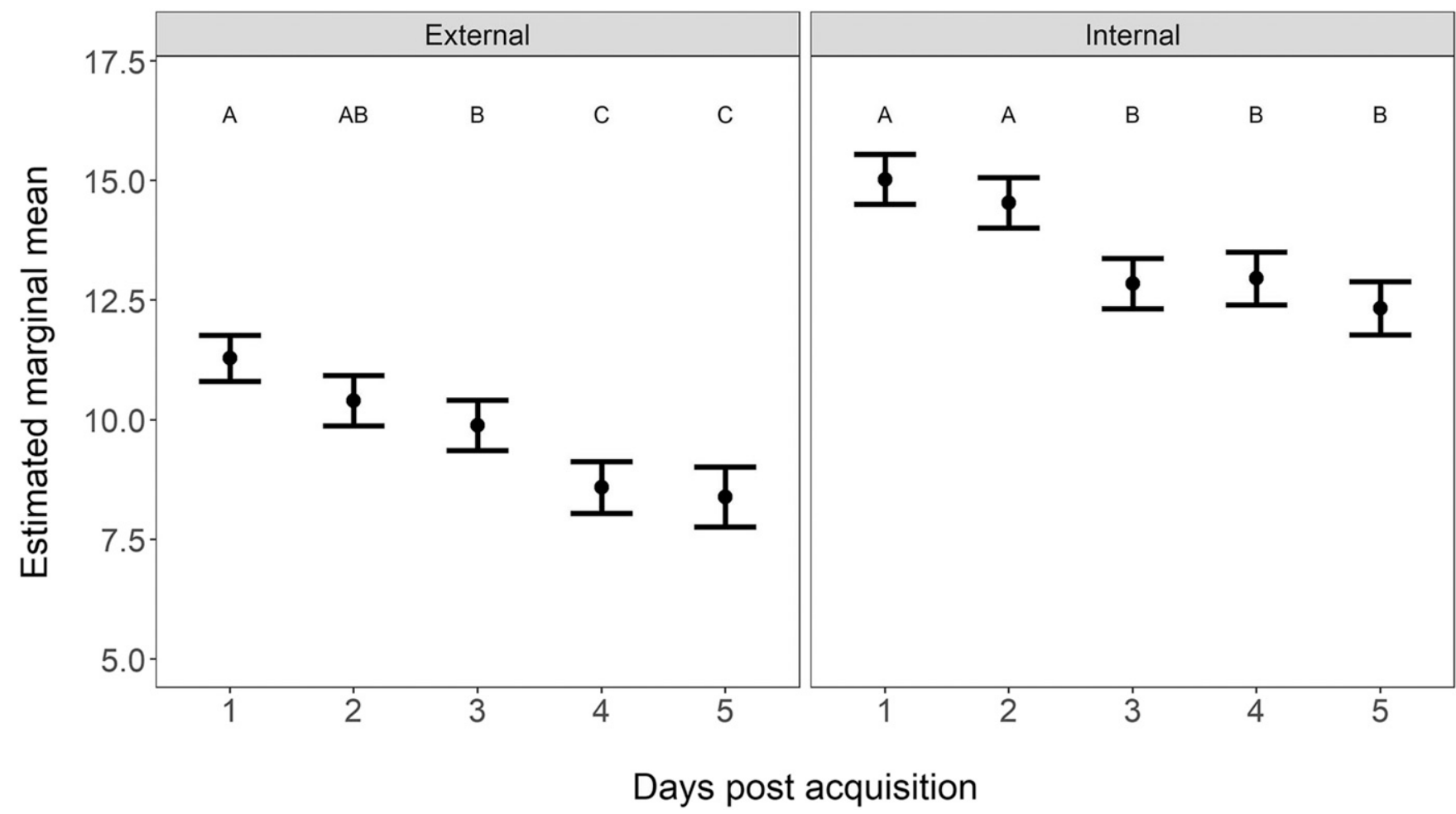

FIGURE 8

Estimated marginal mean $\pm 95 \%$ confidence interval of total Erwinia amylovora colony forming units on external or internal surface of Delia platura over time. Letters indicate significance in bacterial load by day, depicting a gradual decline in both internal and external load over time. Internal loads were higher than external loads on all days as determined by Tukey HSD adjusted pairwise comparisons. 
supporting the theory that dipterans not considered pests of apple can cause losses in an orchard when the proper environmental circumstances align for fire blight outbreaks (Emmett and Baker 1971; Ordax et al. 2015; Slack et al. 2017). We demonstrate the importance of E. amylovora EPS to transmission by showing that flies exposed to nonooze sources of E. amylovora are less likely to successfully initiate infection in saplings than flies exposed to simulated ooze. We hypothesize that ooze aids in adherence of $E$. amylovora to the external surface of the fly and provides a food source that enables establishment of E. amylovora on and inside D. platura. Moreover, the insect-microbe dynamics we present likely enhance the window of infectivity without adversely affecting the fly and can be cautiously generalized across $E$. amylovora strains. We show survival of high bacterial loads within the insect for as long as 7 days, supporting recent assertions that the bacterium can survive internally (Boucher et al. 2019; Ordax et al. 2015).

We conducted transmission experiments using a high density of flies to facilitate comparisons and to account for the inherent randomness of mechanical transmission (Slack et al. 2017; Stewart and Leonard 1916). In the field, D. platura produce three to five generations per growing season, leading to regular fluctuations in population density, and exhibit frequent and widespread swarming behavior, so high densities of this species at one location are not unlikely (Higley and Pedigo 1984; Miller and McClanahan 1960). Filter disk acquisition was used to simulate dispersed or epiphytic populations of E. amylovora that $D$. platura may encounter on an apple sapling or shoot. Rainstorms dissolve ooze, dispersing the bacteria to surrounding leaves and shoots where E. amylovora can survive for variable periods of time depending on environmental conditions (McManus and Jones 1994; Miller 1972). D. platura adults feed on moisture droplets available to them on plant surfaces as well as flower nectar and aphid honeydew (Miller and McClanahan 1960). We have readily observed $D$. platura walking along newly diseased succulent apple shoot growth, probing the surface, and pausing to feed even when E. amylovora ooze is not visibly present. These observations led us to believe that it was possible for flies to acquire E. amylovora from nonooze sources in the field. The severity of E. amylovora infections initiated by $D$. platura subjected to filter disk acquisition were significantly lower than those initiated by flies subjected to ooze acquisition. This result was likely driven by a lower overall incidence of infection initiated by flies fed E. amylovora via filter disks, because samples that did not develop symptoms received a severity of zero. The few positive samples in the filter disk treatment did result in high severity, but the sample size of positive saplings was not large enough to adequately compare with the ooze treatment.

The lower incidence of infection initiated by flies subjected to filter disk acquisition likely resulted from a combination of higher overall dose and higher external dose in flies subjected to ooze acquisition. The relative inefficiency of the fly-mediated transmission mechanism could necessitate bacterial loads surpassing a CFU threshold that acquisition from filter disks or epiphytes does not readily meet (Slack et al. 2017). Although it takes relatively few E. amylovora cells to initiate an infection under controlled conditions (Crosse 1972), flies would need to shed bacteria at temporally and spatially appropriate moments to successfully initiate an infection. Moreover, E. amylovora adhered to the insect surface is considered the primary source of new infections generated by insects (Miller 1972; Parker 1936; Slack et al. 2017) and is transferred onto the plant surface while the insect grooms or at the point of contact between the insect and the plant surface while the insect walks (Miller 1972; Rossmann et al. 2018; Zhukovskaya et al. 2013). Indeed, the likelihood of external contamination decreased by roughly 20 to $60 \%$ over the 5-day

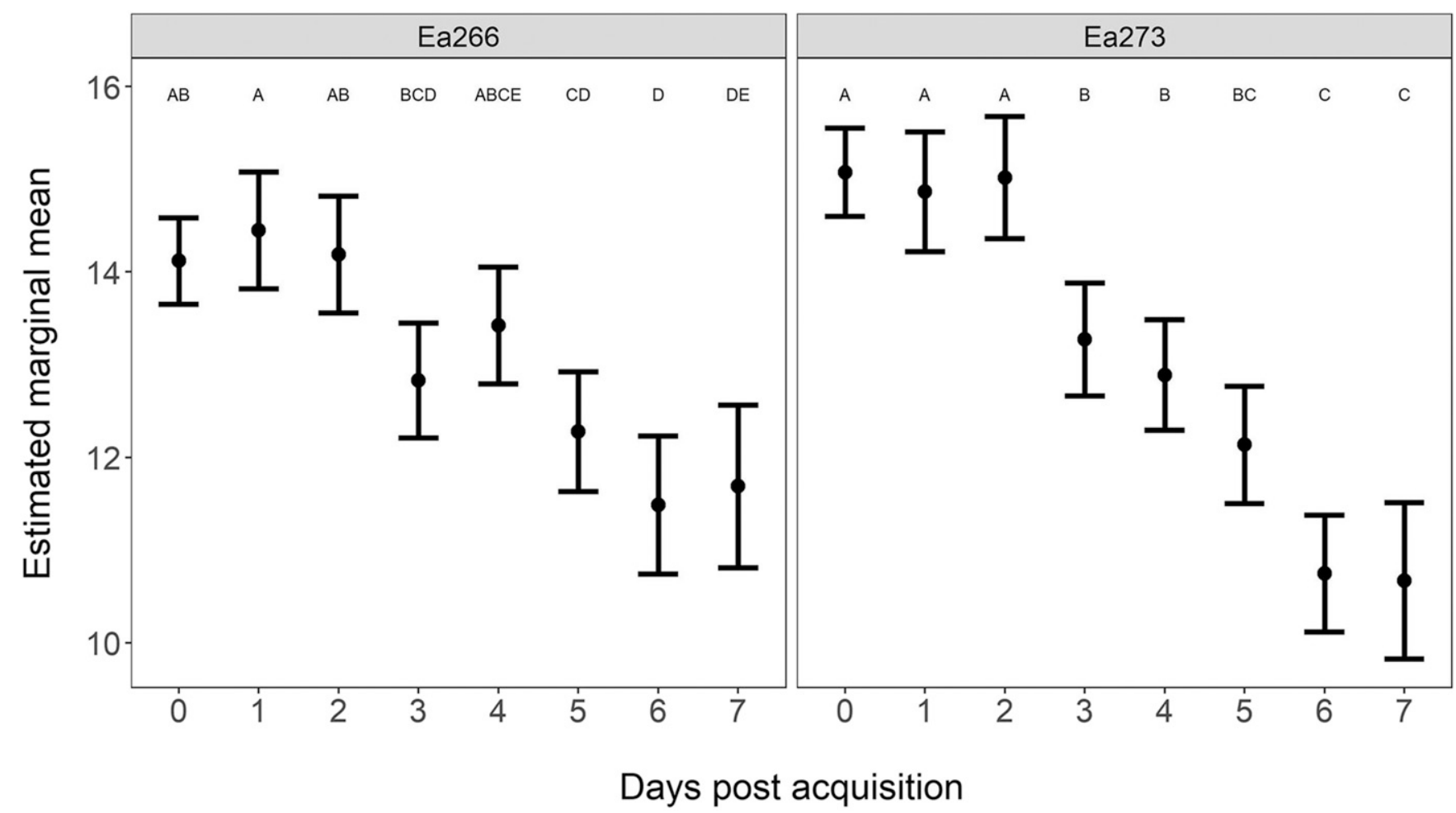

FIGURE 9

Estimated marginal mean $\pm 95 \%$ confidence interval showing change in internal load of Erwinia amylovora in Delia platura over time separated by bacterial strain. Data show pattern change in bacterial load over time within strain. Letters above each time point indicate significant differences between loads from other days within strain as determined by Tukey HSD adjusted pairwise comparisons. 
testing window, indicating frequent shedding from this source. In contrast, internalized bacteria would have to be defecated or regurgitated (Pava-Ripoll et al. 2012), and the likelihood of internal contamination did not change over the testing window despite a depreciation in load. We propose that a low shedding rate is responsible for the observed internal load patterns, making it a less likely source of infection, although additional study is needed to confirm this hypothesis. Thus, higher bacterial loads only available via ooze, especially adhered to the external surface of the fly, likely increase infectivity on a subsequent plant host, whether via direct deposition from the body surface, or less likely via defecation or regurgitation of ingested bacteria.

We demonstrate that flies shed a relatively constant mean daily rate of E. amylovora for at least 4 days following acquisition, and we suggest that flies can initiate new infections for at least that long after acquisition of bacterial loads approximating the levels found in this study. Flies can clear the acquired E. amylovora load, as evidenced by both a lower probability of contamination and shedding over time and by gradually decreasing bacterial loads over time. A possible limitation of the shedding assay is that vials were conducive to growth of other microbes carried by individual flies, and these microbes could have out-competed $E$. amylovora before the vials were tested, resulting in false negatives. Moreover, this assay was conducted in a confined space of $1.5 \mathrm{ml}$, so although our data depict an estimated daily total CFU that a fly sheds, future research should evaluate the relative amount of E. amylovora transferred from internal and external sources during defined behavioral periods. This type of study would better approximate how much bacteria $D$. platura can shed at one moment, providing a more defined idea of how individual and groups of flies contribute to infection success.

There was no effect of E. amylovora strain on shedding, load dynamics, or insect survival. Strains Ea266 and Ea273 had similar mean daily shedding rates and similar internal loads over time. Internal loads of both strains depreciated over time at similar rates. There was no effect of strain on survival, because flies fed either strain did not differ significantly from flies fed a sucrose control. Related bacteria in the Enterobacteriaceae family occasionally exhibit differences in insect-microbe dynamics between strains (Pava-Ripoll et al. 2012), but this was not the case for the dynamics we studied. Strain Ea266 is highly virulent relative to Ea273 (Norelli et al. 1984), and because virulence is correlated with higher EPS production (Ayers et al. 1979), we hypothesized that virulent strains may have higher shedding rates and internal loads over time and lower insect survivability than less virulent strains. We considered the dynamics we tested as the key ones associated with insect-mediated transmission, so based on our data, it is unlikely that virulence would alter the transmission capabilities of insects, although more strains should be studied to confirm the trend we observed.

We provide no evidence that E. amylovora is pathogenic to $D$. platura, because flies fed the bacterium did not differ in likelihood of survival relative to flies fed sucrose. Our findings suggest that molecular interactions between $E$. amylovora and $D$. platura will not negatively influence transmission. Importantly, we cannot rule out active use of $D$. platura by $E$. amylovora as an alternative host due to the presence of nonphytopathogenic type III secretion systems (T3SSs) in the E. amylovora genome (Zhao et al. 2009). T3SSs generally deliver effector proteins to host cells that cause pathogenicity (Preston 2007) but can also mediate interactions between insects and bacterial endosymbionts (Dale et al. 2001). E. amylovora contains three known T3SSs, only one of which is required for pathogenicity in plants (Zhao et al. 2009). The remaining T3SSs are hypothesized to be involved in interactions between E. amylovora and insect hosts (Boucher et al. 2019; Zhao et al. 2009). We suggest here that if the

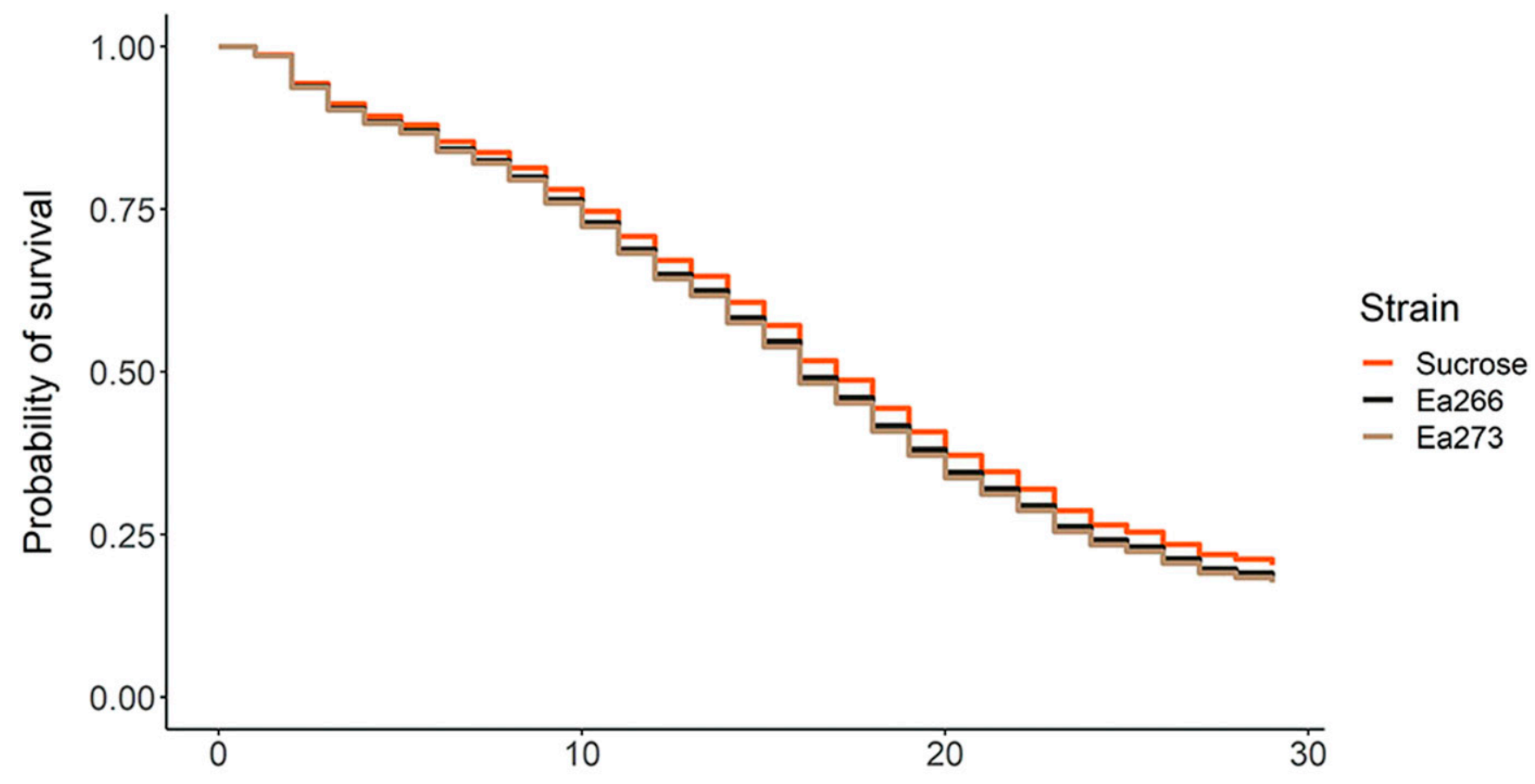

Days post acquisition

FIGURE 10

Probability of survival over time of Delia platura fed one of two Erwinia amylovora strains or a sucrose control as determined by a Cox proportional hazards model. Although survival for flies fed $E$. amylovora regardless of strain was slightly lower than flies fed sucrose, the differences between either strain and the control were not significant $(\mathrm{Ea} 266: \mathrm{z}=0.975, P=0.330 ; \mathrm{Ea273}$ : $\mathrm{z}=1.260, P=0.208)$. 
nonphytopathogenic T3SSs play a role in E. amylovora-D. platura interactions, then that role is not pathogenic and likely involves enhancing bacterial survival in the insect gut, as it does in the Pantoea stewartii-Chaetocnema pulicaria pathosystem in maize (Boucher et al. 2019; Correa et al. 2012), although further study is necessary.

Overall, we add to the growing body of evidence supporting the role of flies in insect-mediated transmission of E. amylovora (Boucher et al. 2019; Ordax et al. 2015; Slack et al. 2017). Although flies were long implicated in the E. amylovora disease cycle, their role was considered less important than other guilds of insects such as pollinating bees that distribute E. amylovora from blossom to blossom or hemipterans that create infection courts through feeding (Parker 1936; Stewart and Leonard 1916). We show that a fly species with worldwide distribution, $D$. platura (Higley and Pedigo 1984), can initiate new infections and that these flies do not need to generate their own entry courts to do so because they can disseminate the bacteria into wounds already present on the plant. Ooze is the most readily available form of $E$. amylovora in the field, and this inoculum source contributes to the establishment of high bacterial loads on and in D. platura. Future research should focus on transmission by D. platura under field conditions, because environmental conditions will drive the success of insect-mediated transmission across temporal and landscape scales. Significant ecological questions remain, especially regarding the relative risk of different forms of plant damage on fly-mediated transmission and the effect of fly density on the likelihood of E. amylovora infection.

\section{ACKNOWLEDGMENTS}

We thank Françoise Vermeylen of the Cornell Statistical Consulting Unit for guidance and instruction throughout data analysis.

\section{LITERATURE CITED}

Ayers, A. R., Ayers, S. B., and Goodman, R. N. 1979. Extracellular polysaccharide of Erwinia amylovora: A correlation with virulence. Appl. Environ. Microbiol. 38:659-666.

Bates, D., Mächler, M., Bolker, B., and Walker, S. 2015. Fitting linear mixedeffects models using lme4. J. Stat. Softw. 67:1-48.

Bellemann, P., Bereswill, S., Berger, S., and Geider, K. 1994. Visualization of capsule formation by Erwinia amylovora and assays to determine amylovoran synthesis. Int. J. Biol. Macromol. 16:290-296.

Bogs, J., Bruchmüller, I., Erbar, C., and Gelder, K. 1998. Colonization of host plants by the fire blight pathogen Erwinia amylovora marked with genes for bioluminescence and fluorescence. Phytopathology 88:416-421.

Bolker, B., and R Development Core Team. 2020. bbmle: Tools for general maximum likelihood estimation. https://cran.r-project.org/package=bbmle.

Boucher, M., Collins, R., Cox, K., and Loeb, G. 2019. Effects of exposure time and biological state on acquisition and accumulation of Erwinia amylovora by Drosophila melanogaster. Appl. Environ. Microbiol. 85: e00726-19.

Brooks, M. E., Kristensen, K., van Benthem, K. J., Magnusson, A., Berg, C. W., Nielsen, A., Skaug, H., Machler, M., and Bolker, B. 2017. glmmTMB balances speed and flexibility among packages for zeroinflated generalized linear mixed modeling. R J. 9:378-400.

Chakrabarti, S., Liehl, P., Buchon, N., and Lemaitre, B. 2012. Infectioninduced host translational blockage inhibits immune responses and epithelial renewal in the Drosophila gut. Cell Host Microbe 12:60-70.

Correa, V. R., Majerczak, D. R., Ammar, E. D., Merighi, M., Pratt, R. C., Hogenhout, S. A., Coplin, D. L., and Redinbaugh, M. G. 2012. The bacterium Pantoea stewartii uses two different type III secretion systems to colonize its plant host and insect vector. Appl. Environ. Microbiol. 78: 6327-6336

Crosse, J. E. 1972. Leaf damage as a predisposing factor in the infection of apple shoots by Erwinia amylovora. Phytopathology 62:176-182.

Crosse, J. E., and Goodman, R. N. 1973. A selective medium for and a definitive colony characteristic of Erwinia amylovora. Phytopathology 63:1425-1426.

Dale, C., Young, S. A., Haydon, D. T., and Welburn, S. C. 2001. The insect endosymbiont Sodalis glossinidius utilizes a type III secretion system for cell invasion. Proc. Natl. Acad. Sci. U.S.A. 98:1883-1888.
Denny, T. P. 1995. Involvement of bacterial polysaccharides in plant pathogenesis. Annu. Rev. Phytopathol. 33:173-197.

Emmett, B. J., and Baker, L. A. E. 1971. Insect transmission of fire blight. Plant Pathol. 20:41-45.

Geider, K. 2004. Twenty years of molecular genetics with Erwinia amylovora: Answers and new questions about EPS-synthesis and other virulence factors. Pages 397-402 in: X International Workshop on Fire Blight 704.

Geier, G., and Geider, K. 1993. Characterization and influence on virulence of the levansucrase gene from the fireblight pathogen Erwinia amylovora. Physiol. Mol. Plant Pathol. 42:387-404.

Goodman, R. N., Huang, J. S., and Huang, P. Y. 1974. Host-specific phytotoxic polysaccharide from apple tissue infected by Erwinia amylovora. Science 183:1081-1082.

Grab, H., Danforth, B., Poveda, K., and Loeb, G. 2018. Landscape simplification reduces classical biological control and crop yield. Ecol. Appl 28:348-355.

Grenier, A. M., Duport, G., Pagès, S., Condemine, G., and Rahbé, Y. 2006. The phytopathogen Dickeya dadantii (Erwinia chrysanthemi 3937) is a pathogen of the pea aphid. Appl. Environ. Microbiol. 72:1956-1965.

Higley, L. G., and Pedigo, L. P. 1984. Seedcorn maggot (Diptera: Anthomyiidae) population biology and aestivation in central Iowa. Environ Entomol. 13:1436-1442.

Hildebrand, E. M. 1939. Studies on fire blight ooze. Phytopathology 29: $142-156$

Kassambara, A., Kosinski, M., and Biecek, P. 2019. survminer: Drawing survival curves using "ggplot2." https://cran.r-project.org/package= survminer.

Koczan, J. M., McGrath, M. J., Zhao, Y., and Sundin, G. W. 2009. Contribution of Erwinia amylovora exopolysaccharides amylovoran and levan to biofilm formation: Implications in pathogenicity. Phytopathology 99: 1237-1244.

Lenth, R. 2020. emmeans: Estimated marginal means, aka least-squares means. https://cran.r-project.org/package=emmeans.

Malnoy, M., Martens, S., Norelli, J., Barny, M., Sundin, G., Smits, T., and Duffy, B. 2012. Fire blight: Applied genomic insights of the pathogen and host. Annu. Rev. Phytopathol. 50:475-494.

McManus, P. S., and Jones, A. L. 1994. Role of wind-driven rain, aerosols, and contaminated budwood in incidence and spatial pattern of fire blight in an apple nursery. Plant Dis. 78:1059-1066.

Miller, L. A., and McClanahan, R. J. 1960. Life history of the seedcorn maggot, Hylemya cilicrura (Rond.) and of H. liturata (Mg.) (Diptera: Anthomyiidae) in southwestern Ontario. Can. Entomol. 92:210-221.

Miller, T. D. 1972. Monitoring the epiphytic population of Erwinia amylovora on pear with a selective medium. Phytopathology 62:1175-1182.

Momol, M. T., Norelli, J. L., Piccioni, D. E., Momol, E. A., Gustafson, H. L., Cummins, J. N., and Aldwinckle, H. S. 1998. Internal movement of Erwinia amylovora through symptomless apple scion tissues into the rootstock. Plant Dis. 82:646-650.

Nadarasah, G., and Stavrinides, J. 2011. Insects as alternative hosts for phytopathogenic bacteria. FEMS Microbiol. Rev. 35:555-575.

Nimtz, M., Mort, A., Domke, T., Wray, V., Zhang, Y., Qui, F., Coplin, D., and Geider, K. 1996. Structure of amylovoran, the capsular exopolysaccharide from the fire blight pathogen Erwinia amylovora. Carbohydr. Res. 287:59-76.

Norelli, J. L., Aldwinckle, H. S., and Beer, S. V. 1984. Differential host $\times$ pathogen interactions among cultivars of apple and strains of Erwinia amylovora. Phytopathology 74:136-139.

Norelli, J. L., Jones, A. L., and Aldwinckle, H. S. 2003. Fire blight management in the twenty-first century: Using new technologies that enhance host resistance in apple. Plant Dis. 87:756-765.

Ordax, M., Piquer-Salcedo, J. E., Santander, R. D., Sabater-Muñoz, B. Biosca, E. G., López, M. M., and Marco-Noales, E. 2015. Medfly Ceratitis capitata as potential vector for fire blight pathogen Erwinia amylovora: Survival and transmission. PLoS One 10:e0127560.

Parker, K. G. 1936. Fire blight: Overwintering, dissemination, and control of the pathogen. N.Y. Agric. Exp. Stn. Mem. 193.

Pava-Ripoll, M., Pearson, R. E. G., Miller, A. K., and Ziobro, G. C. 2012 Prevalence and relative risk of Cronobacter spp., Salmonella spp., and Listeria monocytogenes associated with the body surfaces and guts of individual filth flies. Appl. Environ. Microbiol. 78:7891-7902.

Preston, G. M. 2007. Metropolitan microbes: Type III secretion in multihost symbionts. Cell Host Microbe 2:291-294.

R Core Team. 2019. R: A Language and Environment for Statistical Computing. https://www.r-project.org/.

Rossmann, S., Dees, M. W., Perminow, J., Meadow, R., and Brurberg, M. B. 2018. Soft rot Enterobacteriaceae are carried by a large range of insect species in potato fields. Appl. Environ. Microbiol. 84:e00281-18.

Sebaihia, M., Bocsanczy, A., Biehl, S., Quail, M., Perna, N., Glasner, J., DeClerck, G., Cartinhour, S., Schneider, D., Bentley, S., Parkhill, J., 
and Beer, S. 2010. Complete genome sequence of the plant pathogen Erwinia amylovora strain ATCC 49946. J. Bacteriol. 192:20202021.

Siva-Jothy, J. A., Prakash, A., Vasanthakrishnan, R. B., Monteith, K. M., and Vale, P. F. 2018. Oral bacterial infection and shedding in Drosophila melanogaster. J. Vis. Exp. 135:57676.

Sjulin, T. M., and Beer, S. V. 1978. Mechanism of wilt induction by amylovorin in cotoneaster shoots and its relation to wilting of shoots infected by Erwinia amylovora. Phytopathology 68:89-94.

Slack, S. M., Zeng, Q., Outwater, C. A., and Sundin, G. W. 2017. Microbiological examination of Erwinia amylovora exopolysaccharide ooze. Phytopathology 107:403-411.

Stewart, V. B., and Leonard, M. D. 1915. The role of sucking insects in the dissemination of fire blight bacteria. Phytopathology 5:117-123.

Stewart, V. B., and Leonard, M. D. 1916. Further studies in the role of insects in the dissemination of fire blight bacteria. Phytopathology 6: $152-158$.

Therneau, T. M. 2020. A package for survival analysis in R. https://cran. r-project.org/package $=$ survival.
Thomson, J. L., Yeater, K. M., Zurek, L., and Nayduch, D. 2017. Abundance and accumulation of Escherichia coli and Salmonella typhimurium procured by male and female house flies (Diptera: Muscidae) exposed to cattle manure. Ann. Entomol. Soc. Am. 110:37-44.

van der Zwet, T., and Keil, H. L. 1979. Fire Blight: A Bacterial Disease of Rosaceous Plants. Agricultural Handbook 510. U.S. Department of Agriculture, Washington, DC.

van der Zwet, T., Orolaza-Halbrendt, N., and Zeller, W. 2012. Spread and current distribution of fire blight. Pages 15-36 in: Fire Blight: History, Biology, and Management. American Phytopathological Society, St. Paul, MN.

Webb, D., and Eckenrode, C. 1978. Simplified rearing and bioassay for the seedcorn maggot, Hylemya platura (Meigen). N.Y. Food Life Sci. Bull. 72:1-4.

Wickham, H. 2016. ggplot2: Elegant Graphics for Data Analysis. Springer Verlag, New York, NY.

Zhao, Y., Sundin, G. W., and Wang, D. 2009. Construction and analysis of pathogenicity island deletion mutants of Erwinia amylovora. Can. J. Microbiol. 55:457-464.

Zhukovskaya, M., Yanagawa, A., and Forschler, B. 2013. Grooming behavior as a mechanism of insect disease defense. Insects 4:609-630. 